\title{
A New Path Loss Model for LTE Network to Address Propagation Delay
}

\author{
J. Ramkumar and R. Gunasekaran
}

\begin{abstract}
LTE network has a drastic development in the field of telecommunication broadband wireless networks. LTE uses radio spectrum scenario to provide link path. It uses several propagation models which are available for various networks such as ad hoc networks, cellular networks, etc. Path loss is the significant concept to design and investigate the efficient signal propagation mechanism. In this paper, we propose a new propagation path loss model based on the voice scenario and activating the concept of radio spectrum. In LTE based network, voice and video based services are the emerging concept in radio spectrum path linkage. Proposed propagation path model is simulated mainly on the LTE network scenarios. Here the proposed propagation model is considered with the standard friss propagation model. We have analyzed the delay variation based on the simulation with varying time interval. Propagation model with voice carrier LTE network and radio spectrum technology is used in this paper.
\end{abstract}

Index Terms-Voice bearer, radio spectrum, angle of incidence, uplink scenario.

\section{INTRODUCTION}

Broadband wireless network is emerging drastically in the field of telecommunication in which Long Term Evolution (LTE) Technology is focused here. LTE is the wireless broadband technology to maintain handheld devices and cell phones to support internet access. LTE network provides high speed for data access for mobile phones. It may increase the speed and capacity of a network using different radio interface. LTE technology has been developed with 3GPP (3rd Generation Partnership Project) to meet the rapidly growing user requirements in telecommunication field [1] and [2]. International Telecommunication Unit (ITU) is developing a new technology (LTE Advanced) using mobile communication standard which provides the feature of relay node base station [3].

Path loss occurs when a signal propagates through air medium in NLoS. Path loss is calculated so as to analyze the link establishment in the telecommunication field. Link establishment can be calculated according to the gain and loss of the Transmitter (Tx) / Receiver ( $\mathrm{Rx}$ ) through air medium. Path loss is calculated using propagation losses, absorption, etc [4]. LTE architecture consists of eNodeB (evolved NodeB) and UE (User Equipment) nodes. eNodeB act as a Base Station (BS) which provides several functionalities like selection of MME (Mobile Management Entity), RRM (Radio Resource Management), scheduling, etc. UE act as a

Manuscript received February 13, 2013; revised April 17, 2013.

J. Ramkumar is with the Department of Computer Technology, Anna University, Chennai, India (e-mail: ram.kumar537@gmail.com).

R. Gunasekaran is with the Department of Computer Science and Engineering, Anna University, Chennai, India.
Subscriber Station (SS) such as, mobile phone, data devices, laptops, etc. The architecture of LTE and its components are represented in Fig. 1.

Key elements of LTE networks are,

Mobile Management Entity (MME): Tracking area management, Bearer management, handover, security authentication.

Home Subscriber Server (HSS): Handles the user records and provides authentication.

Evolved Universal Terrestrial Radio Access (E-UTRAN): Uplink and Downlink bearer level admission control, Header compression and user plane ciphering.

Serving Gateway (S-GW): Mobility argument for inter-eNodeB handover and downlink packet buffering.

Packet Gateway (P-GW): IP address allocation, packet filtering and Uplink and Downlink service level rate enforcement.

Policy Charging and Rules Function (PCRF): Resource allocation to UE.

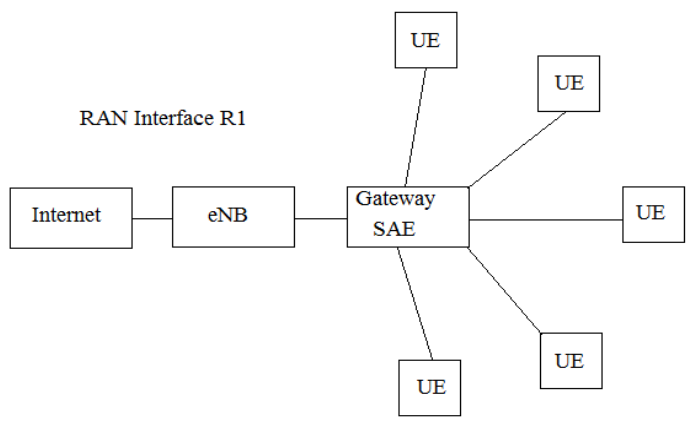

Fig. 1. LTE architecture.

The rest of this paper is organized as follows. Section II outlines the related work, Section III describes terminologies related to radio spectrum, Section IV propose a new propagation model, Section V explains the comparative simulation analysis for both Uplink/Downlink based on LTE network and finally the conclusion is given in Section VI.

\section{RELATED WORK}

Noman Shabbir et al. [5] discusses about the various radio propagation models in LTE network. Various propagation models such as SUI (Stanford University Interim) model, Okumura model, Hata COST 231 model, COST walfisch Ikegami and Ericsson model are compared in two scenarios such as urban and suburban. In [6] and [7] several network propagation models are discussed based on WiMAX environment and several comparisons are made accuracy.

Greg Durgin et al. [8] discusses the propagation path loss is the critical issue for the consumers. Thus the problem of signal propagation through the walls and buildings leads to 
propagation path loss. It develops a path loss model using propagation prediction. Friss H.T., [9] calculated the received power $\left(\mathrm{P}_{\mathrm{r}}\right)$ and transmitted power $\left(\mathrm{P}_{\mathrm{t}}\right)$ known. Power density is determined based on the radiated power and distance in free space medium. Friss transmission discusses the transmitter and receiver antennas as isotropic and path loss is calculated.

Chhaya Dalel et al. [10] predicted accuracy in WiMAX transmission by the proposed COST-231 Hata propogation model and also a comparison is made with the original COST-231 Hata model.

Hata Model. Z. Nadir et al. [11] predicted the mean signal strength in urban areas and median path loss. MSE (Mean Square Error) between measured and predicated path loss is calculated to provide appropriate MSE for radio estimation. Snjezana Rimac-Drlje and Krunoslav Bejuk [12] deal with SUI model to calculate the path loss for three types of terrains like densely populated area, suburban area and rural area. Table 2 represents the parameters in these different terrains. Hemant Kumar Sharma et al [13] discuss about the path loss behavior and propagation models. They Proposed semi deterministic model using walficsh and Ikagami propagation model. Also the comparison between the throughput and jitter with the existing cost 231 propagation models is given.

\section{Propagation PATH LoSS EQUATION}

In a radio propagation pattern, signal undergoes several losses due to various obstacles between BS/SS. Here the NLoS excluding Line of Sight (LoS) is considered. Considering the proposed propagation mechanism to connect NLoS fashion is a challenge to be addressed for a better result.

Signal propagation deals with reflection, diffraction and scattering. Signal propagation between the Tx (BS/SS) and $\mathrm{Rx}(\mathrm{BS} / \mathrm{SS})$ through several obstacles placed between them leads to signal fading. Signal fading can be overcome by reducing the distance between $\mathrm{BS}$ and $\mathrm{SS}$. In free propagation, delay based on the time variation depends on the distance between the BS and SS. Antenna plays a major role to transmit the signal between Tx and Rx.

Considering the $\mathrm{Tx}$ and $\mathrm{Rx}$ separation, received power $P_{r}(d)$ calculated as,

$$
P_{r}(d)=\frac{P_{r} G_{t} G_{r} \lambda^{2}}{(4 \pi)^{2} d^{2} L}
$$

whereas

$P_{t} \rightarrow$ Transmitted power

$G_{t} \rightarrow$ Transmitter antenna gain

$G_{r} \rightarrow$ Receiver antenna gain

$d \rightarrow$ Distance between BS and SS in meters

$L \rightarrow$ System loss factor.

Fading of signal due to obstacles placed between the BS and SS in NLoS results in distortion of propagated signal. Generally fading consists of small scale fading and large scale fading. It can be identified based on the path loss factor. It is calculated as follows [8],

$$
P L(d B)=-10 \log \left[\frac{\lambda^{2}}{(4 \pi)^{2} d^{2}}\right]
$$

whereas,

$P L(d B) \rightarrow$ Path Loss in decibel.

Various other notations in (2) are discussed in (1). Path loss can be calculated based on the received power $P_{r}(d)$ (2). The Signal gets diffracted in all direction when it travels through the air medium with varying reflective index. In (3) Huygens Fresnel principle deals with the problem of signal wave propagation both far field and near field due to diffraction. Far field distance can be calculated as,

$$
d_{0}=\frac{2 D^{2}}{\lambda}
$$

whereas

$D \rightarrow$ Diameter of the antenna

$\lambda \rightarrow$ Wavelength of the radio wave

$d_{0} \rightarrow$ Far field Distance or Fraunhofer distance.

Considering the path loss exponential, Tx and Rx factor and distance path loss function are used. Path loss factor estimates the received power value and $\mathrm{Tx}$ and $\mathrm{Rx}$ distance are considered. Path loss exponential factor is calculated as follows (4),

$$
\overline{P L}(d B)=\overline{P L}(d)+10 n \log \left(\frac{d}{d_{0}}\right)
$$

whereas

$\overline{P L}(d B) \rightarrow$ Path loss Exponent

$d_{\mathrm{o}} \rightarrow$ Close-in-reference distance

$d \rightarrow \mathrm{BS} / \mathrm{SS}$ separation

These are the standardized formulas, to derive the power gain, T-R separation and path loss exponent embedded in the wireless technologies.

\section{Proposed Propagation Model}

Based on the Friss transmission range (1), path loss can be calculated. In NLoS pattern, signal distortion occurs when signal propagates between BS/SS and vice versa. Consider the signal distortion factor, received power $P_{r}(d)$, Path loss $P L(d B)$ and proposed Path loss Exponent $P L_{\text {new }}(d B)$ are calculated. In this new mechanism, uplink/downlink signal propagation is considered based on the height of the Tx/Rx and angle of incidence Fig. 2. The height of the BS $\left(\mathrm{h}_{\mathrm{b}}\right)$ and height of the SS $\left(\mathrm{h}_{\mathrm{S}}\right)$ are discussed in Fig. 5.

Friss H.T. [9] formulated the standard equation through which the power signal can be calculated. Various issues can be solved to meet the utilization of the customer. LTE has focused on the radio spectrum allocation and states the status of data transmission for both voice and video bearer services. Antenna transmission arrangement techniques are broadly classified based on one to one mapping, 1 to $\mathrm{N}$ mapping and $\mathrm{N}$ to $\mathrm{N}$ mapping [5] Data transmitted from eNodeB to UE is referred as Downlink and from UE to eNodeB is referred as Uplink. In Fig. 2 and 3, we have considered both uplink/downlink and $\mathrm{N}$ to $\mathrm{N}$ mapping for our proposed model.

$$
\begin{aligned}
& \text { PLnew }(d B)=84.0 \times 10.0 \times(\log (\text { alpha }) \times \\
& \log (\text { distance }))+(20.0 \times \log (\text { alpha } / \\
& (4.0 \times \text { PI } \times \text { distance })))+\log (f)-C-\log \text { BSSH }
\end{aligned}
$$




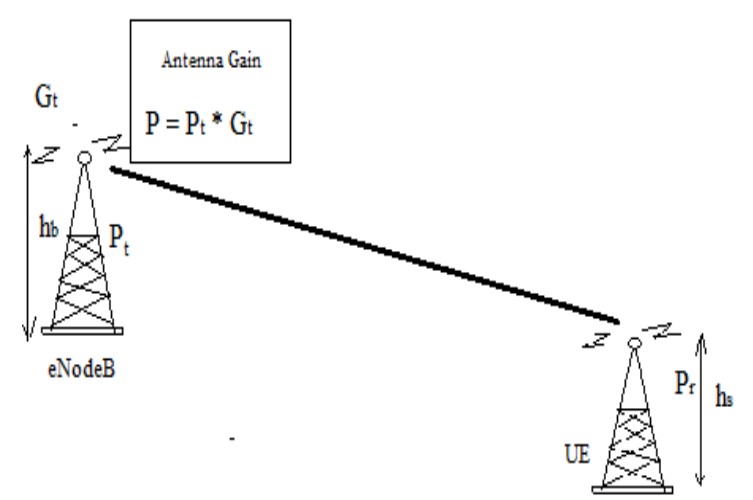

Fig. 2. Propagation link structure.

where

$P L_{\text {new }}(d B) \rightarrow$ Path loss component

Alpha $\rightarrow$ Angle of incidence

$X_{f} \rightarrow$ Frequency factor

$X_{n} \rightarrow$ BS height Correlation

$C \rightarrow$ Shadowing

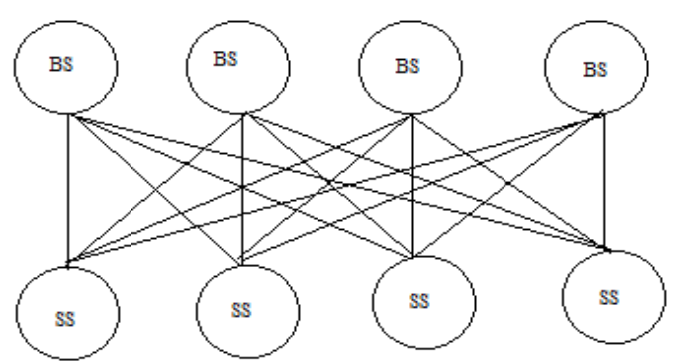

Fig. 3. $\mathrm{N}$ to $\mathrm{N}$ network mapping.

Assigning eNodeB and UE node location based on Mobility model

Bearer channel - type of traffic sent Radio activation and voice type service QoS

Bearer Quality service $=$ General Bearer convert to voice Type

Propagation path loss:

// Initializing the parameters

BS Antenna and SS Antenna Height;

Const. PI $=3.14$;

// Shadowing

Double $c=10$;

$/ / \mathrm{m}$ frequency $=$ carrier frequency

If $\left(\mathrm{m} \_\right.$frequency $\left.>0\right)$

Shadowing $C=1.8 * \log \left(\mathrm{m}_{-} \text {frequency }\right)^{2}-$

$6.4 * \log (m$ frequency $)+c$;

else

NULL;\}

// Radiating Power

Reference distance $D_{0}$;

$\mathrm{D}=$ Distance between eNodeB and UE;

Mobility Model a,b;

If $[(a==12) \&(b==12)]$

\{

Alpha $=\left(h_{b} / C\right)+a+\left(b^{*} h_{s}\right)$

Radiating power $=10 *$ alpha $* \log (D)$;

else

Alpha $=$ NULL;
\}

// Power gain $T x$ and $R x$

$H_{b}=H_{s}=13.82$;

$/ / X_{f}$ and $X_{n}$ referred in (5)

$X_{f}=\log \_\mathrm{BSH}=20.0 * \log \left(h_{b}\right) / 200.0$;

$X_{n}=\log \_\mathrm{BSH}=20.0 * \log \left(h_{b}\right) / 200.0$

(5) Path Loss $=84.0 * 10.0 *(\log ($ alpha $) * \log$

$($ distance $))+(20.0 * \log (\lambda /(4.0 * 3.14 *$

distance) $))+\log \_$f - C_H - log_BSH;

Fig. 4. LTE based propagation path loss algorithm.

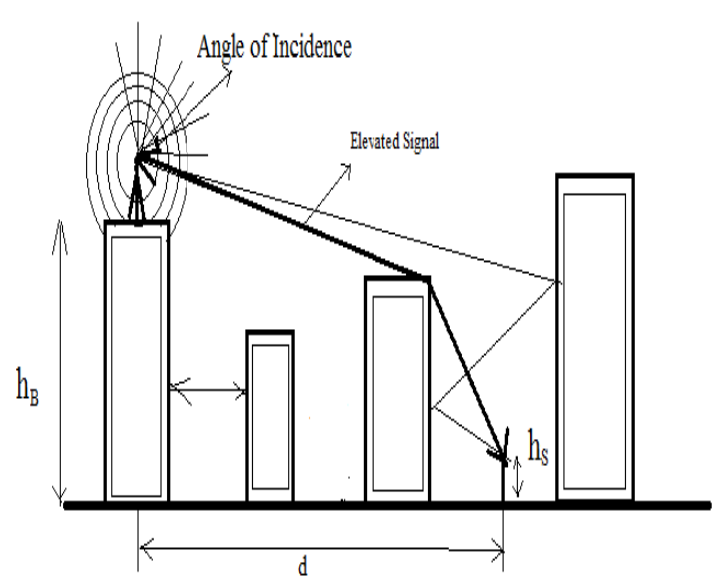

Fig. 5. Diffraction path loss model.

In existing model, only fixed $h_{b}$ and $h_{s}$ is considered, but the new propagation model analyze the path loss by considering the reference distance $\left(\mathrm{d}_{0}\right)$. Considering Fig. 3, we formulate the frequency factor and BS height factor using the $h_{b}$ and $h_{s}$ value.

\section{Simulation}

Considering the LTE network, 4 eNodeB and 4 UE nodes and $\mathrm{N}$ to $\mathrm{N}$ mapping link has been established. It is represented in Fig. 3.

New propagation model is deployed in the LTE network and results are compared with friss path loss propagation model using ns3 simulator. In this simulator, [14] LTE module classes and several mobility model supports are considered and several ns 3 classes are listed below,

Ptr $<$ LteHelper $>$ lteHelper $=$ CreateObject $<$ LteHelper $>()$;

Node container;

MobilityHelper ue1/enB1 mobility;

To activate the radio spectrum and considering the voice data type,

Enum EpsBearer::Qci $q=$ EpsBearer::GBR ${ }_{-} \mathrm{CONV}$ VOICE; EpsBearer bearer $(q)$;

1teHelper->ActivateEpsBearer (ueDevs1, bearer, EpcTft:: Default ());

Based on these LTE based infrastructure for both transmitter and receiver, we have calculated the path loss propagation of delay and standard deviation value as 3.81 and 2.08 .

In Fig. 6, comparisons of both existing Friss propagation model with the proposed propagation model in terms of downlink delay value based on the transmission of both Tx and Rx. 


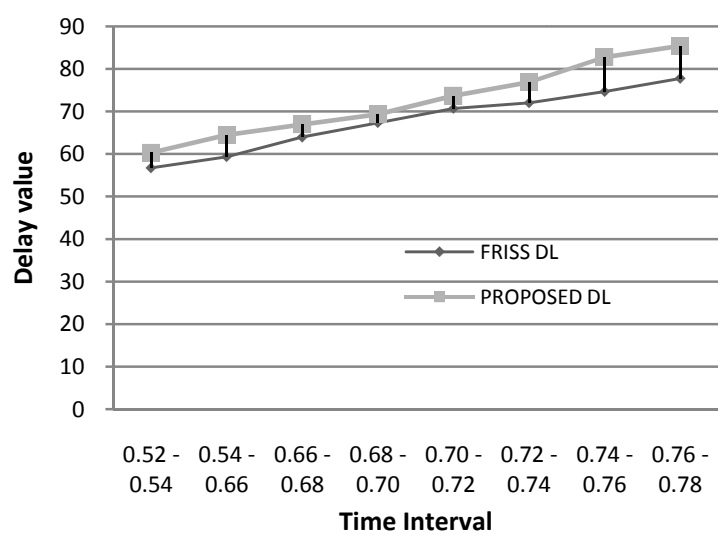

Fig. 6. $T_{x} / R_{x}$ delay variation for downlink.

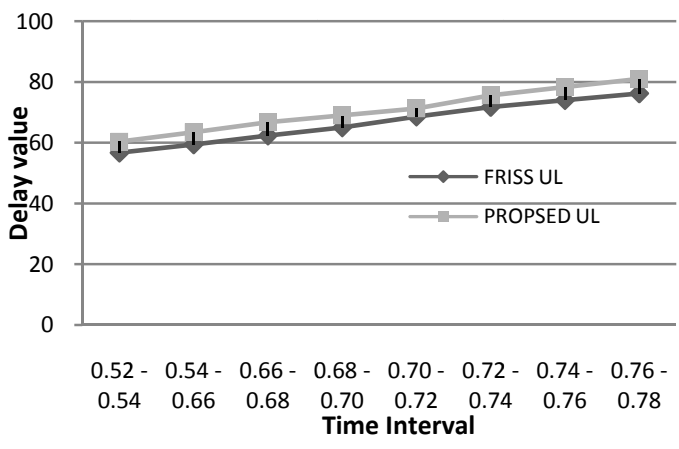

Fig. 7. $T_{x} / R_{x}$ delay variation for uplink.

In Fig. 7, comparisons of both existing friss propagation model with the proposed propagation model in terms of uplink delay value based on the transmission of both $\mathrm{Tx}$ and $\mathrm{Rx}$.

\section{CONCLUSION}

This paper focuses on the propagation path loss to estimate the delay value. As wireless communication technology becomes an essential media for mankind, path loss becomes inevitable. The various propagation models that are proposed till now, mainly focus on the delay value while transmitting data via transmitter and receiver. In this work, a new propagation model based on voice type scenarios is proposed which is suitable for LTE technology. Propagation algorithm considers the different correlation factors for height of both BS and SS. Through ns 3 simulation, we have compared the proposed model with the Friss model. The Simulation result shows that the proposed propagation model reduces the estimated delay value for both uplink and downlink.

\section{ACKNOWLEDGMENT}

This Research was supported by Anna Centenary Research Fellowship (ACRF), Anna University Chennai, India.

\section{REFERENCES}

[1] M. Rao and M. Panchal, "4G wireless technology: A survey paper," in Proc. the National Conference "NCNTE-2012" at Fr. C.R.I.T., Vashi, NaviMumbai, pp. 44- 47, Feb. 2012.

[2] Z. K. Shen, A. Papasakellariou, J. Montojo, D. Gerstenberger, and F. L. $\mathrm{Xu}$, "Overview of 3GPP LTE-Advanced carrier aggregation for $4 \mathrm{G}$ wireless communications," IEEE Communication Magazine, vol. 50, issue. 2, pp. 122-130, Feb. 2012.

[3] A. Ghosh, R. Ratasuk, B. Mondal, N. Mangalvedhe, and T. ThomaS, "Lte-Advanced: Next-Generation wireless broadband technology," IEEE Wireless Communication, vol. 17, issue. 3, pp. 10-22, June 2012

[4] M. S. Rani, S. V. Behara, and K.Suresh, "Comparison of standard propagation model (SPM) and stanford university interim (SUI) radio propagation models for long term evolution (LTE)," International Journal Of Advanced and Innovative Research (IJAIR), pp. 221-228, Nov. 2012.

[5] N. Shabbir, M. T. Sadiq, H. Kashif, and R. Ullah, "Comparison of radio propagation models for long term evolution (lte) network," International Journal of Next-Generation Networks (IJNGN), vol. 3, no. 3, pp. 27-41, Sept. 2011.

[6] D. A. Shalangwa and K. S. Singh, "An empirically base path loss model for GSM fixed wireless access," Journal of Emerging Trends in Computing and Info. Sciences, vol. 2, no. 3, pp. 113-115, Mar 2011.

[7] V. S. Abhayawardhana, I. J. Wassell, D. Crosby, M. P. Sellars, and M. G. Brown, "Comparison of empirical propagation path loss models for fixed wireless access systems," in Proc. IEEE 61 ${ }^{\text {st }}$ Vehicular Technology Conference, 2005. VTC 2005- Spring. 2005, vol. 1, pp. 73-77, June 2005.

[8] G. Durgin, T. S. Rappaport, and H. Xu, "Measurements and models for radio path loss and penetration loss in and around homes and trees at $5.85 \mathrm{GHz}$," IEEE Transactions on Communications, vol. 46, no. 11, pp. 1484-1496, Nov. 1998.

[9] H. T. Friss, "A note on a simple transmission formula," in Proc. the IRE, vol. 34, issue. 5, pp. 254-256, May 1946.

[10] C. Dalela, M. V. S. N. Prasad, and P. K. Dalela, "Tuning of cost-231 hata model for radio wave propagation predictions," in Proc. of Computer Science Conference (CSCP), pp. 255-267, May 2012.

[11] Z. Nadir, N. Elfadhil, and F. Touati, "Path loss determination using Okumura-Hata model and spline interpolation for missing data for Oman," in Proc. The World Congress on Engineering 2008, vol. 1, July 2008

[12] J. Milanovic, S. R. Drlje, and K. Bejuk, "Comparison of propagation models accuracy for WiMAX on $3.5 \mathrm{GHz}$," in Proc. 14th IEEE International Conference on Electronics, Circuits and Systems, 2007, ICECS 2007, pp. 111-114, Dec. 2007.

[13] H. K. Sharma, S. Sahu, and S. Sharma, "Enhanced cost 231 W.I. propagation model in wireless network," International Journal of Computer Applications, vol. 19, no. 6, pp. 36-42, April 2011.

[14] G. Piro, N. Baldo, and M. Miozzo, "An LTE module for the ns-3 network simulator," in Proc. of the 4th International ICST Conference on Simulation Tools and Techniques, SIMUTools '11, pp. 415-422, 2011.

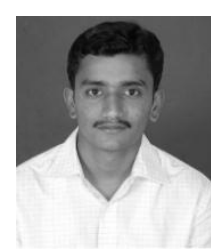

networks.
J. Ramkumar was born in Nov. 1987, he is received the B.Tech. degree in Information Technology from Anna University, Chennai, India, in 2009 and received M.E degree in Computer Science and Engineering, Anna University of Technology, Coimbatore, India. Currently pursuing Ph.D in the faculty of Information and Communication, Anna University, Chennai, India. Research interest includes Wireless Broadband

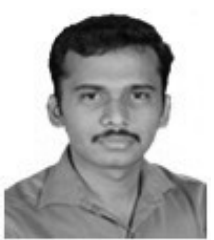

R. Gunasekaran was born on 17 May 1980, he is received the B.Tech. degree in Computer Science and Engineering from University of Madras, Chennai, India, in 2001 and received M.E degree in Computer Science and Engineering from Bharathiyar University, Coimbatore, India. Completed Ph.D under the Department of Computer Science and Engineering, Anna University, Chennai, India in 2010. At present working with Anna University as Associate Professor. Research interest includes Broadband Wireless Networks (3G/4G), Mobile Ad hoc Networks and Network Security. 